Vol. $13 N^{\circ} 1$, pp.53-61 July 1994

Universidad Católica del Norte

Antofag asta - Chile

\title{
PARAMETER-BASED ALGORITHMS FOR APPROXIMATING LOCAL SOLUTION OF NONLINEAR COMPLEX EQUATIONS
}

\author{
I. K. ARGYROS \\ Cameron University, Oklahoma, USA. \\ DONG CHEN \\ University of Arkansas, Arkansas, USA.
}

\begin{abstract}
We study the Ostrowski-Kantorovich convergence for a family of Halley-Werner type iteration methods in the complex plane. We provide an upper error bound for all parameter $\alpha \in[1,2)$. We show that the error bound is a decreasing function of $\alpha$. We prove also that the Halley method has the largest error bound.
\end{abstract}

Key words: Nonlinear equations, Halley-Werner type methods, OstrowskiKantorovich analysis, Upper error bound.

AMS(MOS) Classification: $65 \mathrm{H} 05$ 


\section{Introduction}

The purpose of this paper is the study of Halley-Werner type methods with a parameter $\alpha \in[1,2)$ for solving nonlinear complex equations. This family of methods was proposed by W. Werner in 1980 [5] in the following form:

$$
\begin{gathered}
0=F\left(x_{n}\right)+F^{\prime}\left(x_{n}\right)\left(y_{n}-x_{n}\right), \\
0=F\left(x_{n}\right)+\left[F^{\prime}\left(x_{n}\right)+\frac{\alpha}{2} F^{\prime \prime}\left(x_{n}\right)\left(x_{n+1}-x_{n}\right)\right] \\
+\frac{1-\alpha}{2} F^{\prime \prime}\left(x_{n}\right)\left(y_{n}-x_{n}\right)^{2}
\end{gathered}
$$

for some fixed $\alpha \in \mathbb{R}, n=1,2, \ldots$ For $\alpha=1$ the above method reduces to the famous Halley method or the method of tangent hyperbolas which has a long history [1]. In this paper Ostrowski-Kantorovich type assumptions (similar to the ones already used for Newton's method in [3,4]) are applied to this family of iteration methods. Under this type of assumptions, we establish the existenceuniqueness theorem and give an error bound for $\alpha \in[1,2)$. Finally we show that the error bound of the Halley iteration method is not the best one in this family.

\section{Basic Iteration Characteristics}

First we define an equivalent form for this family:

$$
\begin{gathered}
y_{n}=x_{n}-\frac{F\left(x_{n}\right)}{F^{\prime}\left(x_{n}\right)}, \\
x_{n+1}=-\frac{1}{2} \frac{\frac{F^{\prime \prime}\left(x_{n}\right)}{F^{\prime}\left(x_{n}\right)}\left(y_{n}-x_{n}\right)^{2}}{1+\frac{\alpha}{F^{\prime \prime}\left(x_{n}\right)}}\left(y_{n}-x_{n}\right) .
\end{gathered}
$$

Then we can obtain the following Ostrowski-Kantorovich representation for $F\left(x_{n+1}\right)$. 
PARAMETER-BASED ALGORITHMS

55

Lemma 2.1. Let $F$ be the function in an open convex domain $D$ in $C$ to $C$. Assume that $F$ has second order continuous derivative on $D$. The the following approximation is true for all $n \geq 0$ :

$$
\begin{aligned}
F\left(x_{n+1}\right) & =\int_{0}^{1} F^{\prime \prime}\left(y_{n}+t\left(x_{n+1}-y_{n}\right)\right)(1-t) d t\left(x_{n+1}-y_{n}\right)^{2} \\
& -\frac{\frac{F^{\prime \prime}\left(x_{n}\right)\left(y_{n}-x_{n}\right)^{3}}{2 F^{\prime}\left(x_{n}\right)}\left[(1-\alpha) \int_{0}^{1} F^{\prime \prime}\left(x_{n}+t\left(y_{n}-x_{n}\right)\right)(1-t) d t\right]}{1+\frac{\alpha}{2} \frac{F^{\prime \prime}\left(x_{n}\right)\left(y_{n}-x_{n}\right)}{F^{\prime}\left(x_{n}\right)}} \\
& -\frac{\frac{F^{\prime \prime}\left(x_{n}\right)}{2 F^{\prime}\left(x_{n}\right)}\left(y_{n}-x_{n}\right)^{3} \int_{0}^{1} F^{\prime \prime}\left(x_{n}+t\left(y_{n}-x_{n}\right)\right) t d t}{1+\frac{\alpha}{2} \frac{F^{\prime \prime}\left(x_{n}\right)}{F^{\prime}\left(x_{n}\right)}\left(y_{n}-x_{n}\right)} \\
+ & \frac{\frac{1}{2}\left(y_{n}-x_{n}\right)^{2} \int_{0}^{1}\left[2 F^{\prime \prime}\left(x_{n}+t\left(y_{n}-x_{n}\right)\right)(1-t)-F^{\prime \prime}\left(x_{n}\right)\right] d t}{1+\frac{\alpha}{2} \frac{F^{\prime \prime}\left(x_{n}\right)}{F^{\prime}\left(x_{n}\right)}\left(y_{n}-x_{n}\right)} .
\end{aligned}
$$

Proof: To see this fact, we observe by usual routine that

$$
\begin{aligned}
F\left(x_{n+1}\right)= & F\left(x_{n+1}\right)-F\left(y_{n}\right)-F^{\prime}\left(y_{n}\right)\left(x_{n+1}-y_{n}\right) \\
& +F\left(y_{n}\right)+F^{\prime}\left(y_{n}\right)\left(x_{n+1}-y_{n}\right)
\end{aligned}
$$

and

$$
\begin{aligned}
F\left(y_{n}\right) & +F^{\prime}\left(y_{n}\right)\left(x_{n+1}-y_{n}\right)=F\left(y_{n}\right)-F^{\prime}\left(y_{n}\right) \frac{1}{2} \frac{\frac{F^{\prime \prime}\left(x_{n}\right)}{F^{\prime}\left(x_{n}\right)}\left(y_{n}-x_{n}\right)^{2}}{1+\frac{\alpha}{2} \frac{F^{\prime \prime}\left(x_{n}\right)}{F^{\prime}\left(x_{n}\right)}\left(y_{n}-x_{n}\right)} \\
& =\frac{F\left(y_{n}\right)+\frac{\alpha}{2} \frac{F^{\prime \prime}\left(x_{n}\right)}{F^{\prime}\left(x_{n}\right)}\left(y_{n}-x_{n}\right) F\left(y_{n}\right)-\frac{1}{2} \frac{F^{\prime \prime}\left(x_{n}\right)}{F^{\prime}\left(x_{n}\right)}\left(y_{n}-x_{n}\right)^{2} F^{\prime}\left(y_{n}\right)}{1+\frac{\alpha}{2} \frac{F^{\prime \prime}\left(x_{n}\right)}{F^{\prime}\left(x_{n}\right)}\left(y_{n}-x_{n}\right)}
\end{aligned}
$$

Let us denote the above numerator by $L$. We obtain in turn

$$
F\left(y_{n}\right)=\int_{0}^{1} F^{\prime \prime}\left(x_{n}+t\left(y_{n}-x_{n}\right)\right)(1-t) d t\left(y_{n}-x_{n}\right)^{2},
$$

we now obtain

$$
\begin{aligned}
L= & -\frac{1}{2 F^{\prime}\left(x_{n}\right)}\left[F^{\prime \prime}\left(x_{n}\right)\left(y_{n}-x_{n}\right)^{2} \int_{0}^{1} F^{\prime \prime}\left(x_{n}+t\left(y_{n}-x_{n}\right)\right) d t\left(y_{n}-x_{n}\right)\right. \\
& -\alpha F^{\prime \prime}\left(x_{n}\right)\left(y_{n}-x_{n}\right) \int_{0}^{1} F^{\prime \prime}\left(x_{n}+t\left(y_{n}-x_{n}\right)\right)(1-t) d t\left(y_{n}-x_{n}\right)^{2} \\
& -F^{\prime}\left(x_{n}\right) \int_{0}^{1}\left[2 F^{\prime \prime}\left(x_{n}+t\left(y_{n}-x_{n}\right)\right)(1-t)-F^{\prime \prime}\left(x_{n}\right)\right] d t\left(y_{n}-x_{n}\right)^{2}
\end{aligned}
$$




$$
\begin{aligned}
= & -\frac{1}{2 F^{\prime}\left(x_{n}\right)}\left[(1-\alpha) F^{\prime \prime}\left(x_{n}\right) \int_{0}^{1} F^{\prime \prime}\left(x_{n}+t\left(y_{n}-x_{n}\right)\right)(1-t) d t\left(y_{n}-x_{n}\right)^{3}\right. \\
& +F^{\prime \prime}\left(x_{n}\right) \int_{0}^{1} F^{\prime \prime}\left(x_{n}+t\left(y_{n}-x_{n}\right)\right) t d t\left(y_{n}-x_{n}\right)^{3} \\
& +\frac{1}{2} \int_{0}^{1}\left[2 F^{\prime \prime}\left(x_{n}+t\left(y_{n}-x_{n}\right)\right)(1-t)-F^{\prime \prime}\left(x_{n}\right)\right] d t\left(y_{n}-x_{n}\right)^{2} .
\end{aligned}
$$

Substituting it in the above, we obtain (2.3).

\section{The Ostrowski-Kantorovich Convergence}

Theroem 3.1. Let $F: D \subset C \rightarrow C$, where $C$ is the complex plan and $D$ is an open convex domain. Assume that $F$ has 2 nd order continuous derivatives on $D$, and for given an initial value $x_{0} \in D$ the following Ostrowski-Kantorovich conditions are satisfied:

$$
\begin{gathered}
\left|F^{\prime \prime}(x)\right| \leq M, \quad\left|F^{\prime \prime}(x)-F^{\prime \prime}(y)\right| \leq N|x-y|, \text { for all } x, y \in D, \\
\left|F^{\prime}\left(x_{0}\right)^{-1}\right| \leq \beta, \quad\left|F^{\prime}\left(x_{0}\right)^{-1} F\left(x_{0}\right)\right| \leq \eta .
\end{gathered}
$$

Set $h=K \beta \eta$ and assume that

$$
\begin{gathered}
h \leq \frac{1}{2}, \quad M\left[1+\frac{2 N}{3 M^{2} \beta(2-\alpha)}\right]^{\frac{1}{2}} \leq K, \quad 1 \leq \alpha<2, \\
\frac{S\left(x_{0}, r_{1}\right)}{} \subset D,
\end{gathered}
$$

where $r_{1}$ is the roots of the equation $g(t)=0$, where

$$
g(t)=\frac{K}{2} t^{2}-\frac{1}{\beta} t+\frac{\eta}{\beta}
$$

and

$$
r_{1}=\frac{1-\sqrt{1-2 h}}{h} \eta, r_{2}=\frac{1+\sqrt{1-2 h}}{h} \eta .
$$

The scalar sequences are defined as

$$
\begin{aligned}
s_{n} & =t_{n}-\frac{g\left(t_{n}\right)}{g^{\prime}\left(t_{n}\right)}, t_{0}=0, \\
t_{n+1} & =s_{n}-\frac{1}{2} \frac{\frac{g^{\prime \prime}\left(t_{n}\right)}{g^{\prime}\left(t_{n}\right)}\left(s_{n}-t_{n}\right)^{2}}{1+\frac{\alpha}{2} \frac{g^{\prime \prime}\left(t_{n}\right)}{g^{\prime}\left(t_{n}\right)}\left(s_{n}-t_{n}\right)}, \text { for } n \geq 0 .
\end{aligned}
$$

Then the sequences are well-defined and convergent with $\left\{\underline{x_{n}}\right\},\left\{y_{n}\right\} \in \overline{S\left(x_{0}, r_{1}\right)}$. The limit is the unique solution of the equation $F(x)=0$ in $\overline{S\left(x_{0}, r_{3}\right)}, r_{3} \in\left[r_{1}, r_{2}\right)$. We also have the following error estimates for all $n \geq 0$ :

$$
\left|x_{n}-x^{*}\right| \leq r_{n}-t_{n}
$$




$$
\begin{gathered}
\left|y_{n}-x^{*}\right| \leq r_{1}-s_{n}, \\
r_{1}-t_{n} \leq \frac{\left(1-\theta^{2}\right) \eta}{1-\theta^{3}} \theta^{3^{n}-1}, \text { for all } \alpha \in[1,2),
\end{gathered}
$$

where $\theta=\frac{1-\sqrt{1-2 h}}{1+\sqrt{1-2 h}}$.

Proof: By using induction on $n$, it is enough to show that the following statements are true for all $n \geq 0$ :
$\left(a_{n}\right)$ :
$x_{n} \in \overline{S\left(x_{0}, r_{1}\right)}$,
$\left(b_{n}\right)$ :
$\left(c_{n}\right)$ :
$\left|F^{\prime}\left(x_{n}\right)^{-1}\right| \leq-\frac{1}{g^{\prime}\left(t_{n}\right)}$,
$\left(d_{n}\right):$
$\left|y_{n}-x_{n}\right| \leq s_{n}-t_{n}$,
$\left(e_{n}\right):$
$y_{n} \in \overline{S\left(x_{0}, r_{1}\right)}$,
$\left|x_{n+1}-y_{n}\right| \leq t_{n+1}-s_{n}$.

All the above statements are true for $n=0$ by initial conditions. Then we assume that they are true for a fixed $n$ and all smaller integer values. From $\left(a_{n}\right)$

$$
\begin{aligned}
\left|x_{n+1}-x_{0}\right| & \leq\left|x_{n+1}-y_{n}\right|+\left|y_{n}-x_{n}\right|+\left|x_{n}-x_{0}\right| \\
& \leq\left(t_{n+1}-s_{n}\right)+\left(s_{n}-t_{n}\right)+\left(t_{n}-t_{0}\right) \\
& =t_{n+1}-t_{0} \\
& =t_{n+1} .
\end{aligned}
$$

Hence $\left(a_{n+1}\right)$ is true. Since

$$
\begin{aligned}
\left|F^{\prime}\left(x_{n+1}\right)-F^{\prime}\left(x_{0}\right)\right| & \leq M\left|x_{n+1}-x_{0}\right| \\
& \leq K\left(t_{n+1}-t_{0}\right) \\
& =K t_{n+1} .
\end{aligned}
$$

then

$$
\left|F^{\prime}\left(x_{n+1}\right)^{-1}\right| \leq \frac{1}{\frac{1}{\beta}-K t_{n+1}}=-\frac{1}{g^{\prime}\left(t_{n+1}\right)} .
$$

Thus $\left(b_{n}\right)$ is true. Using (2.3) and taking norms we obtain

$$
\begin{aligned}
\left|F\left(x_{n+1}\right)\right| \leq & \frac{M}{2}\left|x_{n+1}-y_{n}\right|^{2}+\frac{\frac{\left|F^{\prime \prime}\left(x_{n}\right)\right|}{2\left|F^{\prime \prime}\left(x_{n}\right)\right|}\left[(1-\alpha) \frac{M}{2}+\frac{M}{2}\right]\left|y_{n}-x_{n}\right|^{3}}{1-\frac{\alpha}{2} \frac{F^{\prime \prime}\left(x_{n}\right) \mid}{\left|F^{\prime}\left(x_{n}\right)\right|}\left|y_{n}-x_{n}\right|} \\
& +\frac{\frac{N}{6}\left|y_{n}-x_{n}\right|^{3}}{1-\frac{\alpha}{2} \frac{F^{\prime \prime}\left(x_{n}\right) \mid}{F^{\prime}\left(x_{n}\right) \mid}\left|y_{n}-x_{n}\right|} \\
\leq & \frac{K}{2}\left(t_{n+1}-s_{n}\right)^{2}+\frac{\frac{K}{-g^{\prime}\left(t_{n}\right)}\left(s_{n}-t_{n}\right)^{3}(2-\alpha) \frac{K}{4}}{1+\frac{\alpha}{2} \frac{K}{g^{\prime}\left(t_{n}\right)}\left(s_{n}-t_{n}\right)} \\
= & g\left(t_{n+1}\right) .
\end{aligned}
$$


It follows immediately that

$$
\begin{aligned}
\left|y_{n+1}-x_{n+1}\right| & \leq \frac{\left|F\left(x_{n+1}\right)\right|}{\left|F^{\prime}\left(x_{n+1}\right)\right|} \\
& \leq-\frac{g\left(t_{n+1}\right)}{g^{\prime}\left(t_{n+1}\right)} \\
& =s_{n+1}-t_{n+1}
\end{aligned}
$$

and

$$
\begin{aligned}
\left|y_{n+1}-x_{0}\right| & \leq\left|y_{n+1}-x_{n+1}\right|+\left|x_{n+1}-y_{n}\right|+\left|y_{n}-x_{0}\right| \\
& \leq\left(s_{n+1}-t_{n+1}\right)+\left(t_{n+1}-s_{n}\right)+\left(s_{n}-t_{0}\right) \\
& \leq s_{n+1}-t_{0} \\
& =\dot{s}_{n+1}
\end{aligned}
$$

Thus $\left(c_{n+1}\right)$ and $\left(d_{n+1}\right)$ are also true. Moreover from (2.2),

$$
x_{n+2}=y_{n+1}-\frac{1}{2} \frac{\frac{F^{\prime \prime}\left(x_{n+1}\right)}{F^{\prime}\left(x_{n+1}\right)}\left(y_{n+1}-x_{n+1}\right)^{2}}{1+\frac{\alpha}{2} \frac{F^{\prime \prime}\left(x_{n+1}\right)}{F^{\prime}\left(x_{n+1}\right)}\left(y_{n+1}-x_{n+1}\right)},
$$

and by taking norms we shall obtain

$$
\begin{aligned}
\left|x_{n+2}-y_{n+1}\right| & \leq \frac{\frac{1}{2} \frac{\left|F^{\prime \prime}\left(x_{n+1}\right)\right|}{\left|F^{\prime}\left(x_{n+1}\right)\right|}\left|y_{n+1}-x_{n+1}\right|^{2}}{1-\frac{\alpha}{2} \frac{\left|F^{\prime \prime}\left(x_{n+1}\right)\right|}{\left|F^{\prime}\left(x_{n+1}\right)\right|}\left|y_{n+1}-x_{n+1}\right|} \\
& \leq-\frac{1}{2} \frac{\frac{K}{g^{\prime}\left(t_{n+1}\right)}\left(s_{n+1}-t_{n+1}\right)^{2}}{1+\frac{\alpha}{2} \frac{K}{g^{\prime}\left(t_{n+1}\right)}\left(s_{n+1}-t_{n+1}\right)} \\
& =-\frac{1}{2} \frac{\frac{g^{\prime \prime}\left(t_{n+1}\right)}{g^{\prime}\left(t_{n+1}\right)}\left(s_{n+1}-t_{n+1}\right)^{2}}{1+\frac{\alpha}{2} \frac{g^{\prime \prime}\left(t_{n+1}\right)}{g^{\prime}\left(t_{n+1}\right)}\left(s_{n+1}-t_{n+1}\right)} \\
& =t_{n+2}-s_{n+1}
\end{aligned}
$$

Theorefore $\left(e_{n+1}\right)$ is true. Now we are ready to to find the error bound for $r_{1}-t_{n}$. First we set $a_{n}=r_{n}-t_{n}$ and $b_{n}=r_{2}-t_{n}$. Moreover, we notice the facts that

$$
\begin{gathered}
g\left(t_{n}\right)=\frac{K}{2} a_{n} b_{n}, \\
g^{\prime}\left(t_{n}\right)=-\frac{K}{2}\left(a_{n}+b_{n}\right), \\
b_{n}=a_{n}+\frac{\left(1-\theta^{2}\right) \eta}{\theta} .
\end{gathered}
$$


Now by (3.8), we have

$$
a_{n}=\frac{a_{n-1}^{4}+(2-\alpha) a_{n-1}^{3} b_{n-1}}{\left(a_{n-1}+b_{n-1}\right)^{3}-\alpha a_{n-1} b_{n-1}\left(a_{n-1}+b_{n-1}\right)} .
$$

We obtain similarly that

$$
b_{n}=\frac{b_{n-1}^{4}+(2-\alpha) b_{n-1}^{3} a_{n-1}}{\left(a_{n-1}+b_{n-1}\right)^{3}-\alpha a_{n-1} b_{n-1}\left(a_{n-1}+b_{n-1}\right)} .
$$

Taking the ratio of $a_{n}$ and $b_{n}$, we have

$$
\begin{aligned}
\frac{a_{n}}{b_{n}} & =\left[\frac{a_{n-1}}{b_{n}}\right]^{3} \frac{a_{n-1}+(2-\alpha) b_{n-1}}{b_{n-1}+(2-\alpha) a_{n-1}} \\
& =\left[\frac{a_{n-1}}{b_{n-1}}\right]^{3} \frac{\frac{a_{n-1}}{b_{n-1}}+(2-\alpha)}{1+(2-\alpha) \frac{a_{n-1}}{b_{n-1}}}
\end{aligned}
$$

Use the facts that

$$
a_{n-1} \leq b_{n-1}, 1 \leq \alpha<2,
$$

we can easily see that

$$
\frac{a_{n}}{b_{n}} \leq\left[\frac{a_{n-1}}{b_{n-1}}\right]^{3} \leq \ldots \leq\left[\frac{a_{0}}{b_{0}}\right]^{3^{n}}=\theta^{3^{n}}
$$

By solving the above inequality for $a_{n}$, we obtain the error bound

$$
r_{1}-t_{n}=a_{n} \leq \frac{\left(1-\theta^{2}\right) \eta}{1-\theta^{3^{n}}} \theta^{3^{n-1}}
$$

To show uniqueness. let us assume that there exists a second solution $y^{*}$ of equation $F(x)=0$ in $\overline{S\left(x_{0}, r_{3}\right)}$ for $r_{3} \in\left[r_{1}, r_{2}\right)$. Using the approximation

$$
F^{\prime}\left(x^{*}+t\left(y^{*}-x^{*}\right)\right)-F^{\prime}\left(x^{*}\right)=\int_{x_{0}}^{x^{*}+t\left(y^{*}-x^{*}\right)} F^{\prime \prime}(z) d z
$$

and the estimate

$$
\begin{aligned}
& \left|F^{\prime}\left(x_{0}\right)^{-1}\right| \int\left|F^{\prime}\left(x^{*}+t\left(y^{*}-x^{*}\right)\right)-F^{\prime}\left(x_{0}\right)\right| d t \\
\leq & \beta M \int_{0}^{1}\left|x^{*}+t\left(y^{*}-x^{*}\right)-x_{0}\right| d t
\end{aligned}
$$




$$
\begin{aligned}
& \leq \beta M \int_{0}^{1}\left[(1-t)\left|x^{*}-x_{0}\right|+t\left|y^{*}-x_{0}\right|\right] d t \\
& \leq \frac{\beta M}{2}\left(r_{1}+r_{3}\right) \\
& \leq \frac{\beta M}{2}\left(r_{1}+r_{2}\right) \\
& <1 .
\end{aligned}
$$

We deduce that the inverse of

$$
\int_{0}^{1} F^{\prime}\left(x^{*}+t\left(y^{*}-x^{*}\right)\right) d t
$$

exists. From the approximation

$$
F\left(y^{*}\right)-F\left(x^{*}\right)=\int_{0}^{1} F^{\prime}\left(x^{*}+t\left(y^{*}-x^{*}\right)\right) d t\left(y^{*}-x^{*}\right)
$$

we conclude that $x^{*}=y^{*}$. That completes the proof of theorem 3.1.

Some remarks: (a) Under the Ostrowski-Kantorovich assumptions, Gragg and Tapia provided the best error bound for Newton method which can not be improved [2]. That is showed that

$$
r_{1}-t_{n}=\frac{\left(1-\theta^{2}\right) \eta}{1-\theta^{2^{n}}} \theta^{2^{n}-1}, \text { for all } n \geq 0
$$

(b) Similarly, for the Halley method we have the best error bound

$$
r_{1}-t_{n}=\frac{\left(1-\theta^{2} \eta\right.}{1-\theta^{3^{n}}} \theta^{3^{n}-1}, \text { for all } n \geq 0 .
$$

which can also not be improved. Note that we have showed the existence of infinitely many methods for $\alpha \in[1,2)$ where the upper bounds are less than that of the Halley method.

\section{References}

[1] E. Halley, A New Exact and Easy Method of Finding the Roots of equations Generally and that Without any Previous Reduction, Phil. Trans. Roy. London, 18(1694), 134-145.

[2] W. B. Gragg and R.A. Tapia, Optimal Error Bounds for the NewtonKantorovich Theorem, SIAM J. Numer.Anal., 11(1974), 10-13. 
[3] L.V. Kantorovich and G.P. Akilov, Functional Analysis in Normed Spaces, Pergamon Press, New York, 1977.

[4] A.M. Ostrowski, Solution of Equations and Systems of Equations, Academic Press, Third Edition, New York, 1973.

[5] W. Werner, Some Improvements of Classical Iterative Methods for the Solutions of Nonlinear Equations, Lecture Notes in Mathematics, Numerical Solution of Nonlinear Equations, Proceedings, Bremen, 878(1980), 427-440.

Received: December 3, 1992.

Dong Chen

Department of Mathematical Sciences, University of Arkansas,

Fayetteville, Arkansas 72701, USA.

e-mail address: dchen@uafsysb.uark.edu

I.K. Argyros

Department of Mathematics, Cameron University,

Lawton, Oklahoma 73505, USA. 